\title{
Factores asociados al deterioro cognitivo en adultos mayores de la parroquia El Salto - Babahoyo (Ecuador)
}

\author{
Factors associated with cognitive impairment \\ in older adults in the El Salto - Babahoyo parish \\ (Ecuador).
}

Eleana León Tumbaco Universidad Católica de Santiago de Guayaquil (Guayaquil - Ecuador)
Guillermo León Msc Universidad Estatal de Milagro

(Milagro - Ecuador) gfls59@yahoo.es

Revista Cumbres Vol.4 №1

Versión impresa ISSN 1390-9541

Versión electrónica ISSN 1390-3365

http://investigacion.utmachala.edu.ec/revistas/index.php/Cumbres 


\title{
RESUMEN
}

El Deterioro Cognitivo (DC) es una patología crónica que aparece con el envejecimiento, y se asocia a ciertos factores tanto ambientales como personales. En la parroquia El Salto del Cantón Babahoyo, viven un gran número de adultos mayores, y hasta el momento no se han realizado estudios sobre prevalencia de DC ni de factores asociados. El objetivo de este estudio fue determinar la asociación entre los factores socioeconómicos, demográficos, condición laboral y enfermedades crónicas y el DC. El estudio realizado es cuantitativo, correlacional, de corte transversal. Se estudiaron 59 adultos mayores que fueron escogidos de un universo de 540 mediante un muestreo probabilístico aleatorio simple. Se realizó una entrevista y se aplicó una encuesta que analizó los factores que se asocian al DC. Se utilizaron varios instrumentos para consolidar la información y disminuir el margen de error. La información obtenida se procesó en el programa estadístico SPSS utilizando la prueba exacta de Fisher. Los hallazgos indican que los factores que tienen asociación estadísticamente significativa con el DC en los adultos mayores de la parroquia El Salto son la hipertensión arterial, el nivel socioeconómico, el grado de instrucción y el ingreso mensual. Se concluye que el DC en los adultos mayores de la parroquia El Salto está asociado directamente con factores que pueden ser susceptibles de prevención y de intervención, para así contribuir a disminuir o retrasar su aparición.

Palabras clave: Deterioro Cognitivo Leve, Adulto Mayor, Factores asociados, MoCA

\begin{abstract}
Cognitive Impairment (CCD) is a chronic pathology that appears with aging, and is associated with certain environmental and personal factors. In the El Salto del Cantón Babahoyo parish, a large number of elderly people live, and to date no studies have been conducted on the prevalence of CD or associated factors. The objective of this study was to determine the association between socioeconomic factors, demographic, work status and chronic diseases and CD. The study was quantitative, correlational, cross-sectional. We studied 59 older adults who were chosen from a universe of 540 by simple random probabilistic sampling. An interview was conducted and a survey was conducted to analyze the factors associated with CD. Several instruments were used to consolidate the information and reduce the margin of error. The information obtained was processed in the SPSS statistical program using Fisher's exact test. The findings indicate that factors that have a statistically significant association with CD in older adults in the El Salto parish are high blood pressure, socioeconomic status, education level and monthly income. It is concluded that CD in the elderly of the parish El Salto is directly associated with factors that may be susceptible to prevention and intervention, in order to contribute to decrease or delay their appearance.
\end{abstract}

Keywords: Mild Cognitive Impairment, Elderly, Associated Factors, MoCA 


\section{INTRODUCCIÓN}

El envejecimiento es un proceso natural del ser humano que se da a través de todo el ciclo de vida. La calidad de vida y la funcionalidad durante la vejez, se relacionan directamente con las oportunidades y privaciones que se han tenido durante la infancia, la adolescencia y la edad adulta (Buri, 2011).

El envejecimiento de la población ha originado muchos cambios no solo en las estructuras sociales, económicas y culturales de cada país, sino también en las distintas áreas del entorno del adulto mayor (familia, economía, salud, tejido social). Esto ha hecho que diferentes países se preocupen de temas que están relacionados con el trabajo y la jubilación, la calidad de vida, la salud, el involucramiento social y la búsqueda de la seguridad económica de estas personas (Rodríguez, Valderrama, \& Molina, 2010).

"El número de personas que en el mundo rebasa la edad de 60 años, aumentó en el siglo XX a 700 millones en la década del 90; estimándose que para el año 2025 existirán alrededor de 1.200 millones de ancianos" (Alonso et al., 2007).

En Ecuador la población de adultos mayores (AM) representa alrededor del $8 \%$ de la población total, y en los últimos 9 años ha aumentado un $78 \%$ (www.inec.gov.ec 2012). Paralelamente al envejecimiento aumentan las enfermedades crónicas de las cuales el deterioro cognitivo es la más preocupante y costosa, por el daño irreversible que provoca y el alto costo social, familiar y económico que genera.

Las proyecciones señalan que, entre 1980 y el año 2050, la expectativa de vida para las personas mayores de 60 años a nivel mundial aumentará $77 \%$ (Mejía-Arango et al., 2007).

El incremento de la expectativa de vida a nivel mundial, ha traído consigo un aumento en la incidencia de las enfermedades propias de la edad avanzada, de ahí que es muy importante investigar y realizar estudios sobre la aparición de Deterioro Cognitivo (DC), ya que afecta tanto a la calidad de vida de quien lo padece cuanto al núcleo familiar y a la sociedad (Gómez-Viera et al., 2003).

El Deterioro Cognitivo en los AM es un estado en el cual el individuo pierde sus capacidades de razonamiento, entendimiento y de memoria, sin que cumpla con criterios para clasificarlo como demencia. Las personas que lo padecen, suelen tener problemas de convivencia dentro del núcleo familiar, lo cual les lleva a disminuir su calidad de vida.

"El deterioro cognitivo definido como la perdida de funciones cognitivas, depende tanto de factores fisiológicos como ambientales y está sujeto a gran variabilidad interindividual" (Benavides-Caro, 2017).

Existen factores que representan un riesgo significativo en la aparición de DC, relacionados con los estilos de vida, condiciones sociales, y demográficas. Actualmente se ha planteado como factor de riesgo a la insuficiencia renal crónica, aunque los resultados de los estudios son motivo de controversia (Etgen et al., 2012). En pacientes sometidos a hemodiálisis, se estima una prevalencia de deterioro cognitivo entre $28 \%$ y $60 \%$, lo cual representa el doble o más que en pacientes que no reciben este tratamiento renal susti- 
tutivo. Esto es de esperarse, ya que estos pacientes tienen alto riesgo cardiovascular, y hay una estrecha relación entre factores de riesgo cardiovascular y DC (Capote et al., 2016).

Los pacientes que presentan Diabetes Mellitus tipo 2 tienen un volumen cerebral inferior a lo normal, y el DC observado en ellos no sólo está asociado a cambios isquémicos subcorticales, sino también a atrofia cerebral progresiva (Mello et al., 2010).

En un hospital público de Perú se realizó una investigación para determinar asociación entre la diabetes mellitus tipo 2 y el deterioro cognitivo en adultos mayores de 60 años, encontrándose que el 54,5\% de los pacientes con diabetes padecían DC, a diferencia de los que no tenían diabetes apenas el 20,33\% padecían DC (Castro \& Roquelin, 2017).

En el Ecuador se han realizado diversos estudios orientados a investigar el DC y sus factores asociados. En la ciudad de Cuenca en el año 2014 se realizó un estudio transversal en los centros gerontológicos de esa ciudad en el cual encontraron que la prevalencia de DC en adultos mayores de esa población supera el 60\%. El sexo femenino aumenta las probabilidades de padecer, conjuntamente con el nivel de escolaridad bajo o ninguno, encontrándose además algo muy importante que es el hecho de que el DC no se asoció con enfermedades crónicas (Guapisaca, Guerrero, \& Idrovo, 2014).

En el año 2013, en la Universidad de Cuenca se realizó un trabajo sobre DC y factores asociados en adultos mayores jubilados de los servicios sociales del Instituto Ecuatoriano de Seguridad Social (IESS), en el cual constataron que el 35,4\% de los adultos estudiados presentaron DC, y que los factores que más inciden son la edad y la diabetes, y como factor protector el hecho de vivir en el área urbana y no haber fumado (Sánchez, Vásquez, \& Zúñiga, 2013).

En la ciudad de Riobamba en el año 2012 se investigó la relación que existe entre DC y diabetes mellitus, encontrándose que, si bien existe una relación entre estas dos entidades, la asociación no es estadísticamente significativa, pero el hallazgo más importante relacionado a este trabajo es que la hipertensión arterial más Diabetes tiene una relación muy significativa estadísticamente (Astudillo, 2012).

Luego de una exhaustiva revisión bibliográfica sistemática desde 1990 hasta marzo del 2014 realizada por Gracia y colaboradores, en la cual investigaron ocupación laboral y riesgo de deterioro cognitivo y demencia en personas mayores de 55 años, en seis estudios longitudinales se evidenció que los sujetos que mantenían ocupaciones manuales tenían mayor riesgo de desarrollar deterioro cognitivo con respecto a los que han tenido ocupaciones con predominio intelectual (Gracia et al., 2016).

Asimismo en el estudio Paquid (estudio longitudinal con hasta 20 años de seguimiento), realizado en parroquias civiles del sudoeste de Francia en 3.777 adultos mayores de 65 años, encontraron que los sujetos residentes en la comunidad con una ocupación previa intelectual tenían un riesgo de deterioro cognitivo menor ( $\mathrm{HR}=0,8$; IC95\% 0,6-1,0) que aquellos con una ocupación previa no intelectual (Marioni et al., 2014).

La evaluación del estado cognitivo del AM debe ser realizado minucio- 
samente con una historia clínica bien elaborada, orientada a un diagnóstico certero y sus posibles causas. El método de Folstein se utiliza desde 1975 para evaluar el estado cognitivo y se ha convertido en una herramienta que incluso puede predecir la aparición de demencia. Constantemente se han diseñado diferentes instrumentos de fácil aplicación, y con resultados muy satisfactorios.

El Montreal Cognitive Assessment (MoCA), ha mostrado una sensibilidad del $90 \%$ y una especificidad del $87 \%$, utilizando formas básicas que han sido validadas ampliamente. Esta prueba nos permite detectar deterioro cognitivo leve con mucha precisión. La función, concebida como la capacidad para ejecutar actividades básicas, instrumentales y avanzadas de la vida diaria, es un fenómeno complejo en el cual influyen diversos factores como el estado cognitivo, la afectividad, factores sociales, ambientales, económicos, de calidad de vida, etc. La valoración de estas funciones es primordial para establecer un diagnóstico clínico y un pronóstico, como base para decidir el tratamiento y los cuidados de estas personas. Los índices de Katz y de Lawton, son de gran utilidad al momento de evaluar las actividades básicas e instrumentales de la vida diaria respectivamente. Aunque no son los más fidedignos, son los más confiables para orientarnos respecto a la función.

Existen muchos factores que modifican el estado cognitivo y funcional del anciano, entre ellos diversas enfermedades crónicas degenerativas (diabetes mellitus, hipertensión, enfermedad cerebrovascular, cardiopatías, etc), la situación económica (las pensiones que reciben tienen bajo poder adquisitivo, y la necesidad de atención médica e ingesta de medicamentos aumenta constantemente), los factores políticos (falta de programas de ayuda social y de atención en los sistemas de salud), el partido político de turno en el poder, etc. Se dice también que el uso de medicación antihipertensiva está estrechamente vinculado con la aparición de esta patología incluyendo Deterioro Cognitivo Leve (DCL) y Demencia como la Enfermedad de Alzheimer (EA) (Chang-Quan et al., 2011).

Es por estas razones que se hace necesario tener información veraz y fidedigna sobre los factores que intervienen o que se asocian a la aparición de deterioro del estado cognitivo en el adulto mayor, que a su vez nos permita realizar un análisis que conduzca a mejorar los estándares de atención medica que existen en el actual sistema de salud ecuatoriano.

El objetivo del presente estudio fue determinar la asociación que existe entre los factores socioeconómicos, demográficos, condición laboral y enfermedades crónicas y el Deterioro Cognitivo en el adulto mayor de la parroquia "El Salto" de la ciudad de Babahoyo, con la intención de sensibilizar a quienes toman decisiones para que se diseñen mejores programas de atención que beneficien al adulto mayor. 


\section{MATERIALES Y MÉTODOS}

El estudio realizado es cuantitativo, correlacional, de corte transversal. La muestra se seleccionó mediante un muestreo de tipo probabilístico, aleatorio simple. Para determinar el tamaño de la muestra se utilizó la población total de adultos mayores reportada por el departamento de estadística del Subcentro de salud de El Salto (540) con un nivel de confianza del 90\%, un margen de error del 10\%, y un porcentaje estimado del $40 \%$, de donde se extrajeron 59 individuos, a quienes se los contacto personalmente en sus domicilios. Se excluyeron del estudio a aquellas personas que padecían trastornos graves del sensorio, trastornos motrices en miembros superiores, incapacidad mental o física para contestar los cuestionarios, o que no aceptó participar en el estudio

Todos los adultos mayores seleccionados firmaron el consentimiento informado como admisión para participar en el estudio. Se les aplicó el Test de MoCa con la finalidad de detectar DC, y posteriormente se les realizó una entrevista personal para aplicar la ficha de recolección de datos diseñada por el investigador, el mismo que incluye además el Índice de Katz, Índice de Lawton, Índice de Bronfman.

El test de MoCA (Montreal Cognitive Assessment) valora 10 dominios cognitivos, y permite diagnosticar DCL o algún grado de demencia (Rojo-Mota et al., 2013). De acuerdo con el estudio de validación, la sensibilidad y especificidad del test de MoCA para la detección de DC ( $\mathrm{n}=94$ sujetos) fueron $90 \%$ y $87 \%$ respectivamente (Nasreddine et al., 2005).

El Índice De Katz (Valoración de las actividades de la vida diaria), aunque fue diseñado como un índice de rehabilitación, es la escala más utilizada a nivel geriátrico y paliativo. Evalúa el grado de independencia/dependencia de las personas valorando 6 funciones básicas (Ferrín, González, \& Meijide-Míguez, 2011).

El Índice De Lawton (Escala de actividades instrumentales de la vida diaria) evalúa la capacidad para desarrollar tareas que implican el manejo de utensilios habituales y actividades sociales diarias, mediante 8 ítems. Igualmente evalúa el grado de dependencia/independencia en este tipo de actividades (Ferrín et al., 2011).

El Índice del Nivel Socioeconómico De Bronfman evalúa los niveles social y económico de las personas en base a cinco ítems, teniendo 3 categorías de clasificación: bueno, regular y malo (Bronfman et al., 1988).

Una vez recolectados los datos, la información se capturó en una hoja electrónica de Excel para su manejo y almacenamiento, y posteriormente exportada a SPSS versión 20 para su análisis final. Para determinar la asociación a DC se empleó la prueba exacta de Fisher, cuyo valor de $\mathrm{p}<0.050$ nos indica la condición de asociación del factor con el DC. 


\section{RESULTADOS Y DISCUSIÓN}

Se estudiaron 59 adultos mayores, de los cuales 4 fueron excluidos: dos por presentar trastornos graves del sensorio, uno por presentar trastorno en la motricidad de miembros superiores y uno que no aceptó participar en el estudio. De los 55 adultos mayores, el $62 \%$ tuvieron DC, de los cuales el 61,3\% estuvo en el rango de edad entre 65 y 75 años. El 61,3\% perteneció al sexo femenino. 90,3\% tuvo instrucción básica. 54,8\% vive en unión libre, y el 58,1\% tuvo un nivel socioeconómico regular. El 96,8\% tuvo un ingreso mensual menor al salario mínimo vital del país, es decir menos que \$. 354.00 USD. El $90,3 \%$ vive acompañado ya sea por su cónyuge o por sus hijos y en algunos casos por los dos. El 83,9\% no tuvo ocupación y el 54,5\% fue independiente en sus actividades diarias. El 67,7\% no tuvo hábitos tóxicos, es decir no consumía o no había consumido tabaco ni alcohol. El 96,8\% presento hipertensión arterial, el 38,7\%, diabetes, el 9,7\% enfermedad cerebro vascular y el $3,2 \%$ depresión.

Es importante destacar que, del $38 \%$ que no tuvieron DC, el $100 \%$ tuvo un nivel socioeconómico bueno, el $68,4 \%$ un ingreso mensual superior a $\$$. 354.00 USD, y el 73,7\% no tuvo ni ha tenido hábitos tóxicos en el pasado (Tabla 1).

Los factores socioeconómicos y demográficos que se encontraron asociados al DC fueron: la instrucción ( $\mathrm{p}<0.001)$, de la cual la que más predomina es la instrucción básica, el ingreso mensual $(\mathrm{p}<0.001)$ siendo mayoritario el ingreso menor al salario mínimo del país que en el momento de la investigación era de \$. 354.00 y el nivel socioeconómico $(p<0.001)$ que en el presente estudio fue el nivel regular. El resto de variables (edad, sexo, estado civil y estado de convivencia), tienen valores de $\mathrm{p}>0.050$, es decir no se asocian al DC.

Los factores laborales, la dependencia en las actividades básicas e instrumentales de su vida diaria, y los hábitos tóxicos, no tienen ninguna asociación con el DC. De las enfermedades crónicas encontradas en los adultos mayores que presentaron DC, únicamente la hipertensión arterial tiene una asociación estadísticamente significativa (Tabla 2). 
Tabla 1. Análisis factorial en el adulto mayor con y sin DC

\begin{tabular}{|c|c|c|c|}
\hline \multirow[t]{2}{*}{ Variable } & \multirow[t]{2}{*}{ Categoría } & Con DC & Sin DC \\
\hline & & $\%$ & $\%$ \\
\hline \multirow{2}{*}{ Edad } & $65-75 a$ & $61.3 \%$ & $68.4 \%$ \\
\hline & $75-85$ a. & $38.7 \%$ & $31.6 \%$ \\
\hline \multirow{3}{*}{ Sexo } & Masculino & $38.7 \%$ & $36.8 \%$ \\
\hline & Femenino & $61.3 \%$ & $63.2 \%$ \\
\hline & Básico & $90.3 \%$ & $0 \%$ \\
\hline \multirow[t]{2}{*}{ Instrucción } & Secundario & $6.5 \%$ & $73.7 \%$ \\
\hline & Superior & $3.2 \%$ & $26.3 \%$ \\
\hline \multirow{5}{*}{ Estado civil } & Casado & $19.4 \%$ & $31.6 \%$ \\
\hline & Divorciado & $3.2 \%$ & $0 \%$ \\
\hline & Soltero & $3.2 \%$ & $0 \%$ \\
\hline & Unión libre & $54.8 \%$ & $52.6 \%$ \\
\hline & Viudo & $19.4 \%$ & $15.8 \%$ \\
\hline \multirow{2}{*}{$\begin{array}{c}\text { Nivel } \\
\text { socioeconómico }\end{array}$} & Regular & $58.1 \%$ & $0 \%$ \\
\hline & Bueno & $41.9 \%$ & $100 \%$ \\
\hline \multirow{2}{*}{$\begin{array}{l}\text { Ingreso } \\
\text { Mensual }\end{array}$} & $\begin{array}{c}\text { Menos de } 354 \\
\text { USD }\end{array}$ & 96.8 & $31.6 \%$ \\
\hline & $\begin{array}{c}\text { Más de } 354 \\
\text { USD }\end{array}$ & $3.2 \%$ & $68.4 \%$ \\
\hline \multirow{3}{*}{ Con quien vive } & Solo & $9.7 \%$ & $21.1 \%$ \\
\hline & Cónyuge & $54.8 \%$ & $36.8 \%$ \\
\hline & Hijos & $35.5 \%$ & $42.1 \%$ \\
\hline \multirow{3}{*}{ Ocupación } & Ninguna & $83.9 \%$ & $26.3 \%$ \\
\hline & Jubilado & $12.9 \%$ & $52.6 \%$ \\
\hline & $\begin{array}{l}\text { Negocio } \\
\text { propio }\end{array}$ & $3.2 \%$ & $21.1 \%$ \\
\hline \multirow{2}{*}{ Dependencia } & Dependiente & $45.5 \%$ & $15.8 \%$ \\
\hline & Independiente & $54.5 \%$ & $84.2 \%$ \\
\hline \multirow{4}{*}{$\begin{array}{c}\text { Consumo de } \\
\text { alcohol y/o } \\
\text { tabaco }\end{array}$} & Ninguno & $67.7 \%$ & $73.7 \%$ \\
\hline & Tabaco & $25.8 \%$ & $26.3 \%$ \\
\hline & Alcohol & $3.25 \%$ & $0 \%$ \\
\hline & $\begin{array}{l}\text { Alcohol y } \\
\text { Tabaco }\end{array}$ & $3.25 \%$ & $0 \%$ \\
\hline \multirow{4}{*}{$\begin{array}{l}\text { Enfermedades } \\
\text { crónicas }\end{array}$} & HTA & $96.8 \%$ & $36.8 \%$ \\
\hline & Diabetes & $38.7 \%$ & $21 \%$ \\
\hline & $\mathrm{ECV}$ & $9.7 \%$ & $0 \%$ \\
\hline & Depresión & $3.2 \%$ & $0 \%$ \\
\hline TOTAL & & $62 \%$ & $38 \%$ \\
\hline
\end{tabular}


Tabla 2. Factores asociados al DC en el adulto mayor

\begin{tabular}{|c|c|c|}
\hline Variables & Valor p & asociación \\
\hline Edad & 0.421 & NO \\
\hline Sexo & 0.569 & $\mathrm{NO}$ \\
\hline Instrucción & $<0,001$ & SI \\
\hline Estado civil & 0.322 & NO \\
\hline $\begin{array}{c}\text { Nivel } \\
\text { socioeconómico }\end{array}$ & $<0,001$ & SI \\
\hline Ingreso mensual & $<0.001$ & SI \\
\hline Con quien vive & 0.237 & $\mathrm{NO}$ \\
\hline Sin ocupación & 0.062 & NO \\
\hline Dependiente & 0.649 & NO \\
\hline $\begin{array}{l}\text { Consumo de } \\
\text { alcohol y/o } \\
\text { tabaco }\end{array}$ & 0.454 & NO \\
\hline HTA & $<0.050$ & SI \\
\hline Diabetes & 0.162 & $\mathrm{NO}$ \\
\hline ECV & 0.229 & NO \\
\hline Depresión & 0,620 & NO \\
\hline
\end{tabular}

* Test exacto de Fisher

Los adultos mayores son más frágiles después de los 75 años, sin embargo en este estudio la mayoría de los que tienen DC están entre 65 y 75 años de edad, muy similar a la cifra de otros estudios como el de León Arcila realizado en Querétaro México. La mayor parte de los adultos se ubicaron en el estrato socioeconómico regular, probablemente porque se trató de una muestra urbano-marginal, con un ingreso mensual inferior al SMV y que no tienen ninguna ocupación. Si bien el sexo femenino es el más prevalente en- 
tre los adultos que tienen DC, éste no se asocia al mismo, igual que la edad, el estado civil o el hecho de vivir solo.

El no tener ocupación es una variable que no se asocia al DC. Sin embargo estudios más específicos con una muestra mayor, podrían arrojar resultados más precisos para esclarecer este aspecto.

Muchos de estos resultados no coinciden con investigaciones nacionales o internacionales como las de Varela Chávez en Perú en el 2004, Guapizaca en Ecuador en el 2014, o González en el 2013 en la Habana - Cuba, que relacionan el DC con edades mayores a las encontradas en este estudio.

De los factores asociados al DC, el que tuvo mayor significancia estadística fue la Hipertensión arterial. Esto podría explicarse fisiopatológicamente, ya que la HTA causa atrofia de la sustancia blanca cerebral y agrandamiento de los ventrículos laterales. Además produce daños en las arterias cerebrales, causando atrofia subcortical e infartos silentes, lo cual compromete algunos dominios cognitivos, causando a veces daños irreversibles de las funciones intelectuales superiores. Estos resultados concuerdan con el trabajo de González en Cuba, que pone en evidencia que el factor que con más frecuencia se asocia a DC es la hipertensión (51,2\%).

En cuanto al nivel de escolaridad, la instrucción básica se asocia significativamente al DC. Su efecto deletéreo se puede entender si consideramos que un nivel de escolaridad básico se asocia a niveles socioeconómicos bajos, insuficiente nutrición, vivienda y atención en salud. De otra parte los adultos mayores con niveles de escolaridad altos (secundaria y superior), tienen un mejor desarrollo cerebral y un incremento sustancial de la circulación cerebral, ejecutan mejor las pruebas y esto enmascara o retrasa el diagnóstico de DC. Estos resultados concuerdan con los estudios realizados por González en Cuba, de León-Arcila en México y Petersen en Rochester Minesota, quienes reportan asociación significativa entre el grado de instrucción y el DC.

El ingreso mensual por debajo del salario mínimo vital vigente en el país en el momento de la investigación (\$. 354.00), mostro una asociación muy significativa con el DC, y esto podría obedecer a que el poder adquisitivo es muy limitado, y no hay acceso a una buena nutrición o atención de salud, generando consigo detrimento en las actividades mentales superiores y por ende DC que muchas veces puede llegar a la demencia. Son estas mismas razones las que determinaron que el nivel socioeconómico regular se asocie significativamente al DC.

En Inglaterra se encontró que los hombres fumadores son más proclives a presentar DC en la cognición global y la función ejecutiva (Sabia et al., 2012), contrario a los resultados obtenidos en el presente estudio, los mismos que indican que no existe asociación significativa entre DC y hábitos tóxicos entre ellos el consumir tabaco.

Resulta interesante compartir los hallazgos de que la instrucción secundaria o superior, el nivel socioeconómico bueno y el ingreso mensual por encima del salario mínimo vital son los factores que están presentes en la mayoría de los adultos mayores que no tienen DC. Podríamos aventurarnos a considerar como factores protectores de DC en nuestro medio. 


\section{CONCLUSIONES}

Los factores que se asociaron al DC en los adultos mayores de la parroquia El Salto del Cantón Babahoyo, provincia de Los Ríos - Ecuador, fueron el grado de instrucción básico, el ingreso mensual menor al salario mínimo vital (<354.00 USD), el nivel socioeconómico regular y la Hipertensión arterial, los mismos que pueden ser pesquisables tempranamente para un diagnóstico oportuno que nos lleve formular programas de intervención y prevención, que contribuyan a disminuir o retrasar la aparición de esta patología en los adultos mayores.

\section{AGRADECIMIENTO}

El autor expresa su agradecimiento a la Lic. Reyna Tumbaco Salvadores, directora del Subcentro de Salud El Salto, por su permanente colaboración en la recolección de los datos y en la organización de los adultos mayores que fueron objeto de este estudio.

\section{REFERENCIAS BIBLIOGRÁFICAS}

Alonso Galbán, P., Sansó Soberats, F. J., Díaz-Canel Navarro, A. M., Carrasco García, M., \& Oliva, T. (2007). Envejecimiento poblacional y fragilidad en el adulto mayor. Revista Cubana de Salud Pública, 33(1), 0-0.

Astudillo Vallejo, L. (2012). Diabetes mellitus tipo II y deterioro cognitivo leve en pacientes del grupo de jubilados del IESS de Riobamba en el período comprendido entre octubre y noviembre del 2011. Recuperado a partir de http://repositorio.puce.edu.ec/handle/22000/5316

Benavides-Caro, C. A. (2017). Deterioro cognitivo en el adulto mayor. Revista Mexicana de Anestesiología, 40(2), 107-112.

Bronfman, M., Guiscafré, H., Castro, V., Castro, R., \& Gutiérrez, G. (1988). La medición de la desigualdad: una estrategia metodológica, análisis de las características socioeconómicas de la muestra. Arch Invest Med, 19(4), 351-360.

Buri Quizhpe, A. L. (2011). Trastornos emocionales en el adulto mayor en los ancianatos del cantón Loja, mayo 2010-mayo 2011. Recuperado a partir de http://dspace.unl.edu.ec/jspui/handle/123456789/7356

Capote Leyva, E., Casamayor Laime, Z., Castañer Moreno, J., Rodríguez Apolinario, N., Moret Hernández, Y., \& Peña Ruiz, R. (2016). Deterioro cognitivo y calidad de vida del adulto mayor con tratamiento sustitutivo de la función renal. Revista Cubana de Medicina Militar, 45(3), 354-364. 
Castro, R., \& Roquelin, M. (2017). Diabetes mellitus tipo 2 como factor asociado a deterioro cognitivo en pacientes de un hospital público en el Perú. Recuperado a partir de http://repositorio.upao.edu.pe/handle/upaorep/2651

Chang-Quan, H., Hui, W., Chao-Min, W., Zheng-Rong, W., Jun-Wen, G., YongHong, L., ... Qing-Xiu, L. (2011). The association of antihypertensive medication use with risk of cognitive decline and dementia: a meta-analysis of longitudinal studies. International Journal of Clinical Practice, 65(12), 1295-1305. https://doi.org/10.1111/j.1742-1241.2011.02810.x

Ferrín, M. T., González, L. F., \& Meijide-Míguez, H. (2011). Escalas de valoración funcional en el anciano. Galicia Clínica, 72(1), 11-16.

Gómez Viera, N., Bonnin Rodríguez, B. M., Gómez de Molina Iglesias, M. T., Yánez Fernández, B., \& González Zaldívar, A. (2003). Caracterización clínica de pacientes con deterioro cognitivo. Revista Cubana de Medicina, 42(1), 12-17.

Gracia Rebled, A. C., Santabárbara Serrano, J., López Antón, R., Tomás Aznar, C., \& Marcos Aragüés, G. (2016). Ocupación laboral y riesgo de deterioro cognitivo y demencia en personas mayores de 55 años: una revisión sistemática. Revista Española de Salud Pública, 90. Recuperado a partir de http://scielo.isciii.es/scielo.php?pid=S1135-57272016000100501\&script=sci_abstract\&tlng $=$ en

Guapisaca Siguenza, D. J., Guerrero Guevara, J. D., \& Idrovo Toledo, P. A. (2014). Prevalencia y factores asociados al deterioro cognitivo en adultos mayores de los asilos de la ciudad de Cuenca. 2014. Recuperado a partir de http://dspace.ucuenca.edu.ec/handle/123456789/20233

José Adrian Sánchez León, Diana Cecibel Vásquez Bermeo, \& Johanna Elizabeth Zúñiga Peralta. (2013). Prevalencia de Deterioro Cognitivo y factores asociados en adultos mayores jubilados de los servicios sociales del Instituto Ecuatoriano de Seguridad Social, Cuenca 2013. Universidad de Cuenca, Cuenca. Recuperado a partir de http://dspace.ucuenca.edu.ec/ bitstream/123456789/5261/1/MED224pdf.pdf

Marioni, R. E., Proust-Lima, C., Amieva, H., Brayne, C., Matthews, F. E., Dartigues, J.-F., \& Jacqmin-Gadda, H. (2014). Cognitive lifestyle jointly predicts longitudinal cognitive decline and mortality risk. European journal of epidemiology, 29(3), 211-219.

Mejía-Arango, S., Miguel-Jaimes, A., Villa, A., Ruiz-Arregui, L., \& Gutiérrez-Robledo, L. M. (2007). Deterioro cognoscitivo y factores asociados en adultos mayores en México. salud pública de méxico, 49, s475-s481.

Mello, D. C., Lopes, R. M. F., Nascimento, R. F. L., Sartori, F., \& Argimon, I. 
I. I. (2010). El deterioro cognitivo en pacientes ancianos con diabetes mellitus tipo 2 y la identificación de formas de prevención. Neuropsicología, Neuropsiquiatría y Neurociencias, 10(2), 29-42.

Nasreddine, Z. S., Phillips, N. A., Bédirian, V., Charbonneau, S., Whitehead, V., Collin, I., ... Chertkow, H. (2005). The Montreal Cognitive Assessment, MoCA: a brief screening tool for mild cognitive impairment. Journal of the American Geriatrics Society, 53(4), 695-699.

Rodriguez Uribe, A. F., Valderrama Orbegozo, L. J., \& Molina Linde, J. M. (2010). Intervención psicológica en adultos mayores. Psicología desde el Caribe, (25), 246-258.

Rojo-Mota, G., Pedrero-Pérez, E. J., de León, J. M. R.-S., Llanero-Luque, M., \& Puerta-García, C. (2013). Cribado neurocognitivo en adictos a sustancias: la evaluación cognitiva de Montreal. Revista de Neurología, 56(3), 129-136.

Sabia, S., Elbaz, A., Dugravot, A., Head, J., Shipley, M., Hagger-Johnson, G., ... Singh-Manoux, A. (2012). Impact of smoking on cognitive decline in early old age: the Whitehall II cohort study. Archives of General Psychiatry, 69(6), 627-635. https://doi.org/10.1001/archgenpsychiatry.2011.2016 